\title{
Long-term Single-centre Outcomes After Proctocolectomy With Ileoanal Anastomosis for Paediatric Ulcerative Colitis
}

\author{
liris Nyholm, ${ }^{a}$ Maria Hukkinen, ${ }^{a}$ Antti Koivusalo, ${ }^{a}$ Laura Merras-Salmio, \\ Kaija-Leena Kolho, ${ }^{c}$ Risto J. Rintala, ${ }^{a}$ Mikko P. Pakarinena
}

\begin{abstract}
a Section of Paediatric Surgery, Children's Hospital, Helsinki University Hospital, Helsinki, Finland 'bection of Paediatric Gastroenterology, Children's Hospital, Helsinki University Hospital, Helsinki, Finland 'Section of Paediatric Gastroenterology, Tampere University Hospital and University of Tampere, Tampere, Finland
\end{abstract}

Corresponding author: Mikko P. Pakarinen MD, PhD, Helsinki University Hospital, Children's Hospital, P.0. Box 281, 00029 HUS, Helsinki, Finland. Tel.: +358-50-4272981; fax: +358-9-47175737; email: mikko.pakarinen@hus.fi

Conference presentation: European Congress of Pediatric Surgery, EUPSA, Paris, 2018.

\begin{abstract}
Background and Aims: Childhood-onset ulcerative colitis [UC] requires total colectomy in onequarter of patients at some point of their disease. The study objective was to evaluate long-term outcomes after proctocolectomy with ileoanal anastomosis [IAA] for paediatric UC.

Methods: Medical records of all children undergoing proctocolectomy with IAA for UC during 1985-2016 in Helsinki University Hospital were retrospectively assessed. Data on disease history, diagnostic and operative details, occurrence of surgical complications, functional outcome, postoperative diagnosis of Crohn's disease [CD] and pouch failure were collected. Risk factors for IAA failure were analysed with Cox regression.

Results: Of 87 patients, 85 [98\%] had UC and 2 [2\%] inflammatory bowel disease unclassified [IBD-U] preoperatively. Altogether $66 \%$ underwent two-stage and $34 \%$ underwent three-stage procedures. During $7.8[4.1-14.5]$ years' follow-up, nine [10\%] patients were diagnosed with postoperative CD. Postoperative leakages $[n=8,9 \%]$ and strictures $[n=10,11 \%]$ were equally common, whereas fistulas $[78 \%$ vs $9 \%, p<0.001]$ and abscesses [ $56 \%$ vs $14 \%, p=0.009]$ were more frequent among patients with later $\mathrm{CD}$ diagnosis. At latest follow-up, eight [9\%] patients had been converted to a permanent ileostomy and others reported daytime stooling frequency of 5 [4-7] and 0.5 [0-1] at night. $\mathrm{CD}$ diagnosis (hazard ratio $[\mathrm{HR}]=23.3, p=0.005$ ), postoperative abscesses $[\mathrm{HR}=16.3, p=0.013]$ and fistulas [HR $=20.9, p=0.007]$ as well as three-stage surgery [ $p=0.018]$ increased risk for ileostomy. Conclusions: For paediatric UC, long-term surgical and functional outcomes after proctocolectomy with IAA are reassuring. Need for three-stage surgery and occurrence of postoperative fistulas and abscesses, but not leakages or strictures, associate with postoperative $\mathrm{CD}$ diagnosis and the risk for ileostomy.
\end{abstract}

Key Words: Colitis; ulcerative; proctocolectomy; restorative; Crohn's disease

\section{Introduction}

Ulcerative colitis $[\mathrm{UC}]$ is a disease with rapidly increasing incidence and tendency towards a more aggressive course in the quarter of patients presenting with childhood-onset UC. ${ }^{1}$ Despite constantly evolving medical management options, ${ }^{2} 24 \%$ of patients still need surgery at some point of their lifelong disease. ${ }^{3}$ Proctocolectomy with ileoanal anastomosis [IAA] and pouch reconstruction have become the procedure of choice for UC in both children and adults, and carries 
good long-term outcomes. ${ }^{4,5}$ Nevertheless, postoperative pouch failure occurs in $5-10 \%$ of adult patients and in up to $14 \%$ of children, and frequently leaves the patient with a permanent ileostomy. ${ }^{5-15} \mathrm{On}$ the other hand, the long-term functional outcomes among children are similar to those of adults, , ,6,16-18 and after IAA surgery, most children with UC are able to wean off corticosteroid and immunomodulatory medications as well as to experience significant improvements in quality of life, growth, and nutritional status. ${ }^{10,18}$

Despite improving understanding of inflammatory bowel disease [IBD] characteristics and careful patient selection for IAA procedures, a preoperative diagnosis of UC will later turn out to be Crohn's disease $[\mathrm{CD}]$ in a minority of patients. Postoperative CD occurs in $2-4 \%$ of adults ${ }^{13-15}$ and in some series in up to $30 \%$ of paediatric patients, ${ }^{8-10,18-21}$ and relates to significantly worse functional outcomes as well as increasing the risk for pouch failure. ${ }^{13-15,22}$ In addition, up to half of patients undergoing IAA require reoperations for either surgical complications or bowel obstruction. ${ }^{7,8,23}$ Postoperative abscesses, fistulas, and strictures occur in up to onethird of paediatric patients, and some studies have found such complications to increase the risk for ending up with an ileostomy. ${ }^{5-10,17,18}$ Preoperative identification of patients at risk for CD or pouch failure remains very challenging, and studies evaluating their predictors among children are frequently limited by their short follow-up periods and ${ }^{16,18}$ small patient samples, ${ }^{17}$ as well as involvement of young adults who may present with different disease characteristics and outcomes compared with children. ${ }^{6,8-10}$ The purpose of this study was to evaluate single-centre long-term outcomes after proctocolectomy with IAA for paediatric UC, focusing on pouch failure and its predictors, postoperative $\mathrm{CD}$ occurrence, surgical complications, and functional outcomes.

\section{Materials and Methods}

\subsection{Patients and data collection}

All patients who underwent proctocolectomy with IAA in Helsinki University Children's Hospital [Helsinki, Finland] between 1985 and 2016 were included in this study. Patients' medical records were retrospectively reviewed for diagnostic details, disease history, operative details, the occurrence of postoperative strictures, leakages, abscesses, fistulas, and relaparotomies, as well as for postoperative diagnosis of CD and pouch failure. Data on bowel function and pouchitis episodes were collected from medical records at time of latest follow-up or before abandoning IAA. Follow-up time extended from surgery to latest follow-up in the Helsinki University Hospital.

In accordance with Finnish legislation, no approval by an ethical committee or informed consent is required for registry-based studies done without contacting the study subjects.

\subsection{Outcome variables}

The primary outcome measure pouch failure was defined as presenting with an ileostomy at the latest follow-up. The diagnosis of postoperative $\mathrm{CD}$ was based on a comprehensive evaluation of the histopathological, endoscopic, and clinical disease characteristics by experienced paediatric or adult gastroenterologists, surgeons, and pathologists. The diagnostic criteria for CD included typical clinical symptoms, intestinal histopathology and endoscopic findings or exceptionally severe perianal disease with recurrent fistula formation in the absence of surgical complications. The occurrence of postoperative leakages, abscesses, fistulas, and strictures, as well as postoperative haemorrhages and occlusions requiring laparotomy, were recorded. Anastomotic stenosis dilated at least twice was considered a stricture. Inflammation in the pouch was defined as pouchitis if the patient was symptomatic and had therefore been prescribed antibiotics during the year preceding the follow-up. Occurrence of faecal accidents was registered as severe incontinence, and scarce or rare staining of underwear was considered mild incontinence. Patientreported defaecation frequencies were registered.

\subsection{IAA surgery}

Proctocolectomy and IAA with placement of a diverting ileostomy were primarily performed during the same surgery unless the patient's condition warranted three-stage surgery with a separate colectomy. Transanal mucosectomy was started about $1 \mathrm{~cm}$ above the dentate line, and the distal ileum was handsewn to the anal canal after creation of a stapled ileal pouch. A few patients, operated mostly during the beginning of the inclusion period, underwent a straight IAA which was performed in a similar fashion but without pouch formation. Stapled anastomosis without mucosectomy was performed in three patients among whom the distal ileum did not reach the perineum even after extensive mobilisation and division of the ileocolic artery. Routine investigations before surgery have included gastroscopy, ileocolonoscopy, and capsule endoscopy since December 2002, and magnetic resonance imaging enterography in selected cases after January 2009. After IAA, patients were regularly followed up by the operating paediatric colorectal surgeon, a paediatric gastroenterologist, and a stoma nurse. Endoscopic examination of pouch and distal ileum was routinely performed 6-12 months postoperatively and when indicated by clinical symptoms. Oral sodium chloride substitution and loperamide medication were used to limit high stooling frequency during the early postoperative period. After the age of 18 years, patients were transitioned to adult gastroenterology services at individually tailored age.

\subsection{Statistics}

Data were analysed using RStudio [version 1.0.143.0] with Epi, epitools, survival, and survminer packages. Categorical variables are expressed as frequencies and continuous variables as medians and interquartile ranges. Fisher's exact test was used comparing frequencies of categorical variables. Continuous data were analysed with the Mann-Whitney U test [Wilcoxon rank sum test] and KruskalWallis or one-way analysis of variance [ANOVA] in case of three or more groups. Predictors for anastomosis survival were analysed with the Cox proportional hazards regression model by restricting the follow-up time at 10 years, in order to limit bias caused by patients' different follow-up times. Three $p$-values were calculated with likelihood, Wald, and log-rank tests, and the chosen method had the least statistical significance. Significance level was determined as 0.05 .

\section{Results}

\subsection{Patient characteristics}

We identified in total 87 patients, of whom two [2\%] had a diagnosis of unclassified inflammatory bowel disease [IBD-U] and the remainder of UC before surgery. Median age at diagnosis was 11.8 years and at surgery was 14.2 years [Table 1]. Half of the patients $[n=43$ ] were female. Median follow-up time from surgery was 7.8 years. There was no operation-related mortality.

Joint disease, including arthralgia, arthropatia, and arthritis, was the most common associated disease, occurring in $14 \%$ of patients. Other associated diseases were primary sclerosing cholangitis, gallstones, asthma, pancreatitis, and thromboembolic complications, occurring each in less than $7 \%$ of patients [Table 1]. 
Table 1. Patient characteristics.

\begin{tabular}{|c|c|c|c|c|}
\hline & All & UC & Later CD diagnosis & $p$-Values \\
\hline Patients, $n[\%]$ & 87 [100] & $78[90 \%]$ & $9[10 \%]$ & \\
\hline Female, $n[\%]$ & $43[49 \%]$ & $40[51 \%]$ & $3[33 \%]$ & 0.484 \\
\hline Age at diagnosis, [years] & $11.8[8.9-13.3]$ & $11.7[8.0-13.3]$ & $11.9[10.0-12.2]$ & 0.517 \\
\hline Age at surgery [years] & $14.2[12.3-16.1]$ & $14.3[12.4-16.1]$ & $13.5[11.9-14.8]$ & 0.681 \\
\hline Follow-up time [years] & $7.8[4.1-14.5]$ & $6.9[3.9-13.0]$ & $13.6[11.2-17.2]$ & 0.021 \\
\hline Time from surgery to CD diagnosis [years] & - & - & $6.4[5.3-11.0]$ & - \\
\hline IAA abandoned, $n[\%]$ & $8[9 \%]$ & $1[1 \%]$ & $7[78 \%]$ & $<0.001$ \\
\hline Type of anastomosis, $n[\%]$ & & & & 1.000 \\
\hline Pouch & $76[87 \%]$ & $68[87 \%]$ & $8[89 \%]$ & \\
\hline Straight & $11[13 \%]$ & $10[13 \%]$ & $1[11 \%]$ & \\
\hline Stages, $n[\%]$ & & & & 0.265 \\
\hline 2 & $57[66 \%]$ & $53[68 \%]$ & $4[44 \%]$ & \\
\hline 3 [separate colectomy] & $30[34 \%]$ & $25[32 \%]$ & $5[56 \%]$ & \\
\hline \multicolumn{5}{|l|}{ Indication for surgery, $n[\%]$} \\
\hline Refractory disease & $48[55 \%]$ & $43[55 \%]$ & $5[56 \%]$ & 1.000 \\
\hline Steroid-dependent disease & $32[37 \%]$ & $30[38 \%]$ & $2[22 \%]$ & 0.727 \\
\hline Toxic megacolon & $3[3 \%]$ & $2[3 \%]$ & $1[11 \%]$ & 0.282 \\
\hline Other & $4[5 \%]$ & $3[4 \%]$ & $1[11 \%]$ & 0.359 \\
\hline \multicolumn{5}{|l|}{ Associated diseases, $n[\%]$} \\
\hline Joint disease & $12[14 \%]$ & $10[13 \%]$ & $2[22 \%]$ & 0.605 \\
\hline Primary sclerosing cholangitis [PSC] & $5[6 \%]$ & $5[6 \%]$ & $0[0 \%]$ & 1.000 \\
\hline Asthma & $5[6 \%]$ & $4[5 \%]$ & 1 [11\%] & 0.429 \\
\hline Pancreatitis & $4[5 \%]$ & $3[4 \%]$ & $1[11 \%]$ & 0.359 \\
\hline Gallstone disease & $3[3 \%]$ & $2[3 \%]$ & $1[11 \%]$ & 0.282 \\
\hline Thromboembolic complications & $3[3 \%]$ & $3[4 \%]$ & $0[0 \%]$ & 1.000 \\
\hline
\end{tabular}

Continuous data are given as median with interquartile range; $p$-values from the Mann-Whitney U test, Fisher exact test or Kruskal-Wallis test are reported.

UC, ulcerative colitis; CD, Crohn's disease; IAA, ileo-anal anastomosis.

\subsection{Operative details}

The most common indication for proctocolectomy and IAA was UC refractory to medical therapy [ $55 \%$, Table 1]. Other indications included steroid-dependent disease with or without refractory disease [ $37 \%, n=32]$, complications related to steroid use [ $2 \%, n=2]$, pyoderma gangraenosum associated with UC $[1 \%, n=1]$, and delayed growth $[1 \%, n=1]$. Toxic megacolon leading to emergency colectomy had occurred in three patients $[3 \%]$.

The majority of patients underwent IAA with a pouch reconstruction $[87 \%]$, and a straight anastomosis [SIAA] was performed in $13 \%$ [Table 1]. Ten out of 11 SIAA patients were operated before 1991. The IAA was handsewn in 84 [97\%] patients and stapled in three $[3 \%]$ patients.

\subsection{Postoperative complications}

Overall, 48 [55\%] patients presented with a postoperative complication, of which intestinal occlusions requiring relaparotomy were the most common [Table 2]. Leakages were registered in eight [9\%], strictures in the anastomosis or pouch in 10 [11\%], abscesses in 18 [18\%], and fistulas in 14 [16\%] patients. Median time from the primary surgery to leakage was 112 days [81-137], and strictures were diagnosed a median 64 [19-550] days, abscesses a median $2.3[0.2-5.3]$ years, and fistulas a median $4.8[0.4-7.3]$ years after surgery. One leakage occurred after colectomy but before IAA reconstruction, whereas other leakages occurred after IAA surgery. Three or more postoperative abscesses occurred in four patients, of whom three were later diagnosed with CD. Three or more fistulas were observed in two patients, both of whom were later diagnosed with CD.

Altogether 37 [43\%] patients underwent 59 relaparotomies after IAA surgery. In addition to adhesive bowel obstruction with or without strangulation [ $n=33,56 \%$ of reoperations], surgery was performed for leakages $[n=7,12 \%]$, abscesses or fistulas $[\mathrm{n}=5$, $8 \%$ ], redo IAA [ $n=3,5 \%$ ], postoperative haemorrhage [ $n=3,5 \%$ ], non-mechanical occlusion $[n=3,5 \%]$, and other indications $[n=5$, $8 \%$ ]. Indications for redo IAA after short-term ileostomy were prolonged abscess or fistula formation in the pouch $[n=2]$ and diarrhoea with straight anastomosis $[n=1]$. All patients with redo IAA remained UC diagnosis in follow-up.

\subsection{Postoperative Crohn's disease}

After IAA surgery, CD was diagnosed in nine patients, representing $10 \%$ of the study sample. Median time from surgery to CD diagnosis was 6.4 [5.3-11.0] years. In addition to typical clinical findings, the diagnosis of CD was based on intestinal histopathology in five patients [ $56 \%$ ]. In two cases [22\%], CD diagnosis was supported by endoscopic findings although histology was not entirely definitive of $\mathrm{CD}$. In the remaining two patients [22\%], CD diagnosis was based on strong clinical evidence consisting of refractory perianal disease with recurrent fistula formation associated with frequent pouchitis episodes, although histological and endoscopic findings remained inconclusive. Any postoperative surgical complication had been registered in eight [89\%] patients with later $\mathrm{CD}$ diagnosis, compared with $21[27 \%$ ] of UC patients [ $p=0.011]$. In particular, fistulas and abscesses were more frequent in patients with CD [Table 2].

\subsection{Pouch failure and its risk factors}

The IAA had been abandoned in eight [9\%] patients presenting with a permanent ileostomy at latest follow-up. Seven [88\%] out of these eight patients had been diagnosed with CD postoperatively. On the other hand, pouch failure occurred in seven out of nine [78\%] patients later diagnosed with $\mathrm{CD}[p<0.001$ compared with UC 
Table 2. Surgical complications according to later Crohn's disease [CD] diagnosis.

\begin{tabular}{|c|c|c|c|c|}
\hline & All patients & Ulcerative colitis & Later $\mathrm{CD}$ diagnosis & $p$-value \\
\hline Patients, $n[\%]$ & $87[100 \%]$ & $78[90 \%]$ & $9[10 \%]$ & \\
\hline Follow-up time [years] & $7.8[4.1-14.5]$ & $6.9[3.9-13.0]$ & $13.6[11.2-17.2]$ & 0.021 \\
\hline All complications, $n[\%]$ & $48[55 \%]$ & $41[53 \%]$ & $7[78 \%]$ & 0.178 \\
\hline Stricture, $n[\%]$ & $10[11 \%]$ & $7[9 \%]$ & $3[33 \%]$ & 0.275 \\
\hline Leakage, $n[\%]$ & $8[9 \%]$ & $7[9 \%]$ & $1[11 \%]$ & 1.000 \\
\hline Fistula, $n[\%]$ & $14[16 \%]$ & $7[9 \%]$ & $7[78 \%]$ & $<0.001$ \\
\hline Abscess, $n[\%]$ & $16[18 \%]$ & $11[14 \%]$ & $5[56 \%]$ & 0.009 \\
\hline Occlusion requiring laparotomy, $n[\%]$ & $25[29 \%]$ & $22[28 \%]$ & $3[33 \%]$ & 0.712 \\
\hline Haemorrhage requiring laparotomy, $n[\%]$ & $3[3 \%]$ & $2[3 \%]$ & $1[11 \%]$ & 0.282 \\
\hline Other complications requiring laparotomy, $n[\%]$ & $7[8 \%]$ & $6[8 \%]$ & $1[11 \%]$ & 0.548 \\
\hline
\end{tabular}

Follow-up time given as median with interquartile range and complications as frequencies; $p$-values calculated with Mann-Whitney U test and Fisher's exact test.

patients]. In the Cox proportional hazards regression model with a maximum follow-up time of 10 years, later CD diagnosis as well as postoperative fistulas and abscesses were significant risk factors for pouch failure; age at diagnosis or at surgery, sex, anastomosis type, strictures, leakages, or need of relaparotomy were unrelated to pouch failure [Table 3 and Figure 1]. Notably, six out of the eight patients $[75 \%]$ with pouch failure had undergone three-stage surgery $[p=0.018$ when compared with patients undergoing two-stage IAA]. All patients who ended up with an ileostomy within 10 years had undergone three-stage IAA as well as having experienced at least one postoperative complication [ $p=0.007]$.

\subsection{Functional outcome and pouchitis episodes}

In the whole sample, median defaecation frequency at latest follow-up was five times a day and once a night [Table 4]. Among patients later converted to ileostomy, the corresponding frequencies were 9.3 [7.510] per day and two [1-2.6] per night, whereas those who retained intestinal continuity presented with defaecation frequencies 5 [4.5-7.5] times a day and $0.5[0-1.5]$ a night $[p=0.004$ and 0.007 , respectively]. Data on continence and pouchitis episodes were reliably documented in 63 patients [ $72 \%$ of the total sample and $83 \%$ out of the 76 patients with a pouch]. Based on their medical records, $59 \%$ [ $n=37]$ were fully continent and $40 \%[n=25]$ had rare or scarce staining. Only one patient $[2 \%]$ had severe incontinence. No pouchitis during the preceding year had occurred in $43 \%$ [ $n=27]$ of patients, occasional pouchitis had been recorded in $38 \%[n=24]$, and there were recurrent pouchitis episodes in $19 \%$ [ $n=12]$. No association between the number of annual pouchitis episodes and daily bowel function frequency was observed [one-way ANOVA, $\mathrm{F}=2.00, p=0.101$ ].

\section{Discussion}

In our study, the long-term outcomes after proctocolectomy with ileoanal anastomosis were reassuring, with $91 \%$ of patients retaining their intestinal continuity and presenting with good functional outcomes after a median follow-up of 8 years, extending beyond 15 years in quarter of the patients. In line with earlier studies, postoperative CD was clearly the most significant risk factor for pouch failure. Reflecting CD characteristics or preoperative uncertainty of the diagnosis, postoperative fistulas and abscesses, three-stage procedure, and overall postoperative complication rate were associated with an increased incidence of pouch failure.

Pouch failure after IAA has been reported to occur in $2-14 \%$ of paediatric patients in the long term..$^{5-10}$ Previous studies among children have found postoperative $\mathrm{CD}$, perianal disease, pelvic
Table 3. Predictors of pouch failure assessed with Cox proportional hazards regression.

\begin{tabular}{|c|c|c|c|c|}
\hline & Coefficient & HR & $95 \% \mathrm{CI}$ & $p$-Value \\
\hline Age at IBD diagnosis, [per year] & 0.51 & 1.66 & $0.99-2.75$ & 0.052 \\
\hline Age at surgery, [per year] & 0.17 & 1.18 & $0.84-1.65$ & 0.338 \\
\hline Female sex & -0.44 & 0.65 & $0.11-3.88$ & 0.632 \\
\hline Pouch vs straight anastomosis & -0.10 & 0.91 & $0.10-8.19$ & 0.930 \\
\hline Three- vs two-stage surgery ${ }^{a}$ & 21.63 & - & - & 1.000 \\
\hline Surgical complications ${ }^{\mathrm{b}}$ & 19.95 & - & - & 1.000 \\
\hline Stricture & 0.14 & 1.15 & $0.13-10.37$ & 0.903 \\
\hline Leakage & 0.75 & 2.12 & $0.24-18.94$ & 0.536 \\
\hline Fistula & 3.04 & 20.86 & $2.33-187.1$ & 0.007 \\
\hline Abscess & 2.79 & 16.27 & $1.81-145.9$ & 0.013 \\
\hline Occlusion requiring & 1.59 & 4.90 & $0.81-29.5$ & 0.080 \\
\hline \multicolumn{5}{|l|}{ laparotomy } \\
\hline $\begin{array}{l}\text { Haemorrhage requiring } \\
\text { laparotomy }\end{array}$ & 1.61 & 4.98 & $0.55-44.74$ & 0.200 \\
\hline Relaparotomy & 1.76 & 5.82 & $0.65-52.15$ & 0.100 \\
\hline Later CD diagnosis & 3.15 & 23.34 & $2.59-210.7$ & 70.005 \\
\hline
\end{tabular}

Variables calculated from univariable analysis with 10 years' maximum follow-up time. Three $p$-values were calculated with likelihood, Wald and logrank tests, and the chosen method had the least statistical significance.

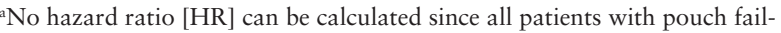
ure within 10 years had undergone three-stage surgery.

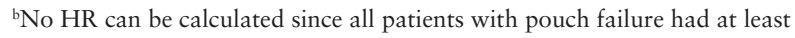
one surgical complication.

$\mathrm{CD}$, Crohn's disease; CI, confidence interval; HR, hazard ratio; IBD, inflammatory bowel disease.

sepsis, chronic pouchitis, and poor functional outcomes to be risk factors for ending up with an ileostomy. ${ }^{8}$ Our overall pouch failure rate of $9 \%$ compares favourably with earlier studies. ${ }^{8-10,20}$ Further, our results demonstrate that with appropriate patient selection and prompt management of postoperative complications, only postoperative $\mathrm{CD}$ diagnosis remains a significant risk factor for pouch failure during follow-up. Higher pouch failure rates observed among patients with postoperative fistulas and abscesses reflect the frequency of such complications in $\mathrm{CD} .{ }^{22}$ Accordingly, perianal disease, frequently encountered in CD patients, and female sex, have been reported to increase the risks for pouch failure in children. ${ }^{8,19,23}$

Differentiating UC from CD is particularly challenging among children. ${ }^{24}$ Compared with adults, children present more frequently with total colonic $\mathrm{CD}$, which often clinically mimics paediatric UC..$^{25}$ On the other hand, children with UC may present with clinical characteristics overlapping with $\mathrm{CD}$, such as rectal sparing, segmental 
A

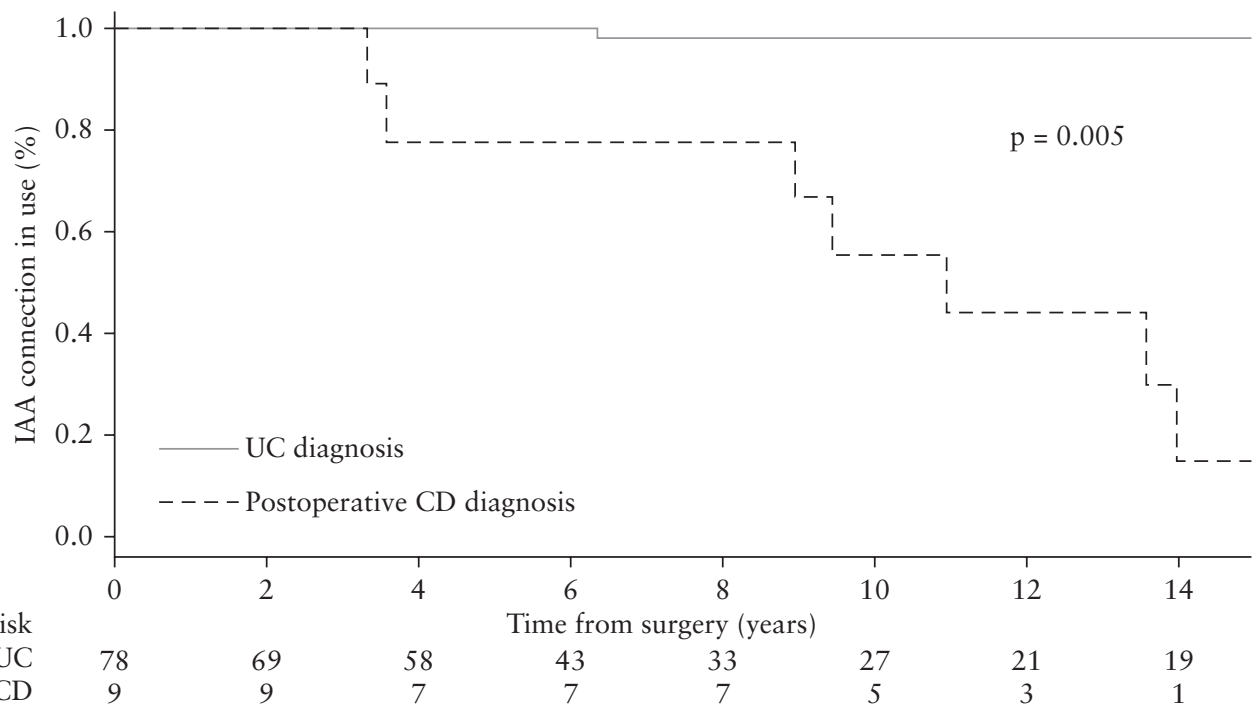

B

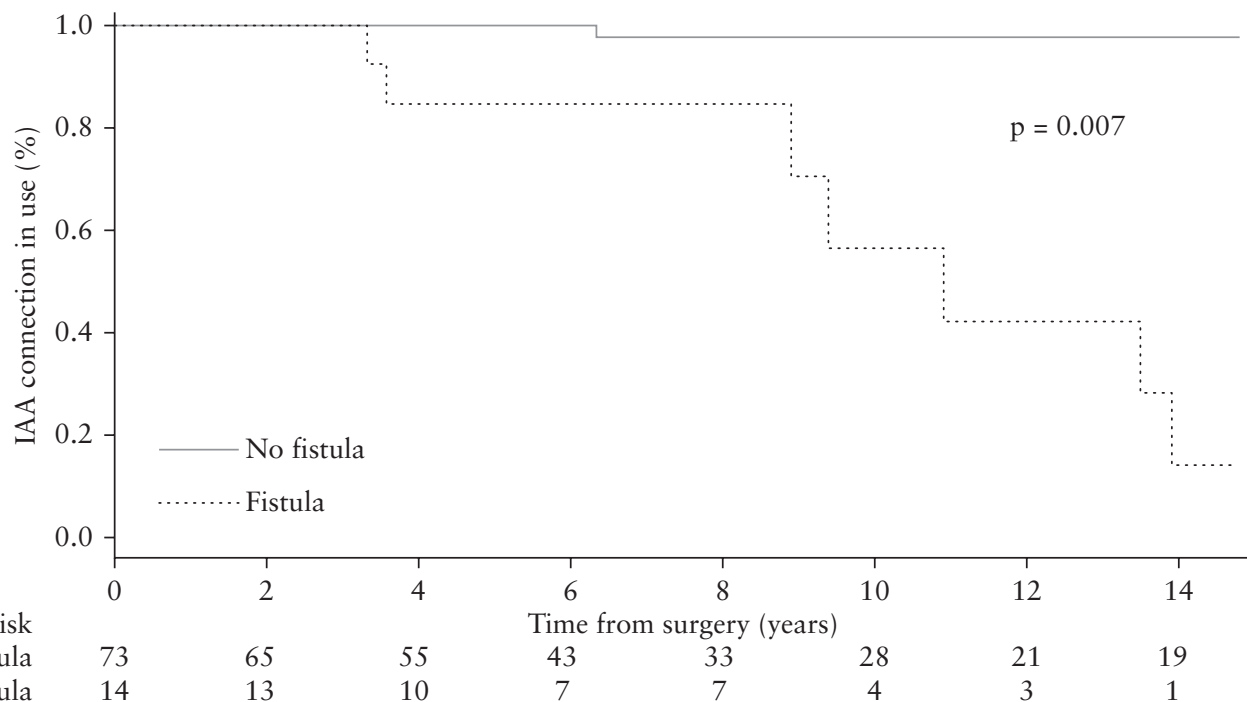

C

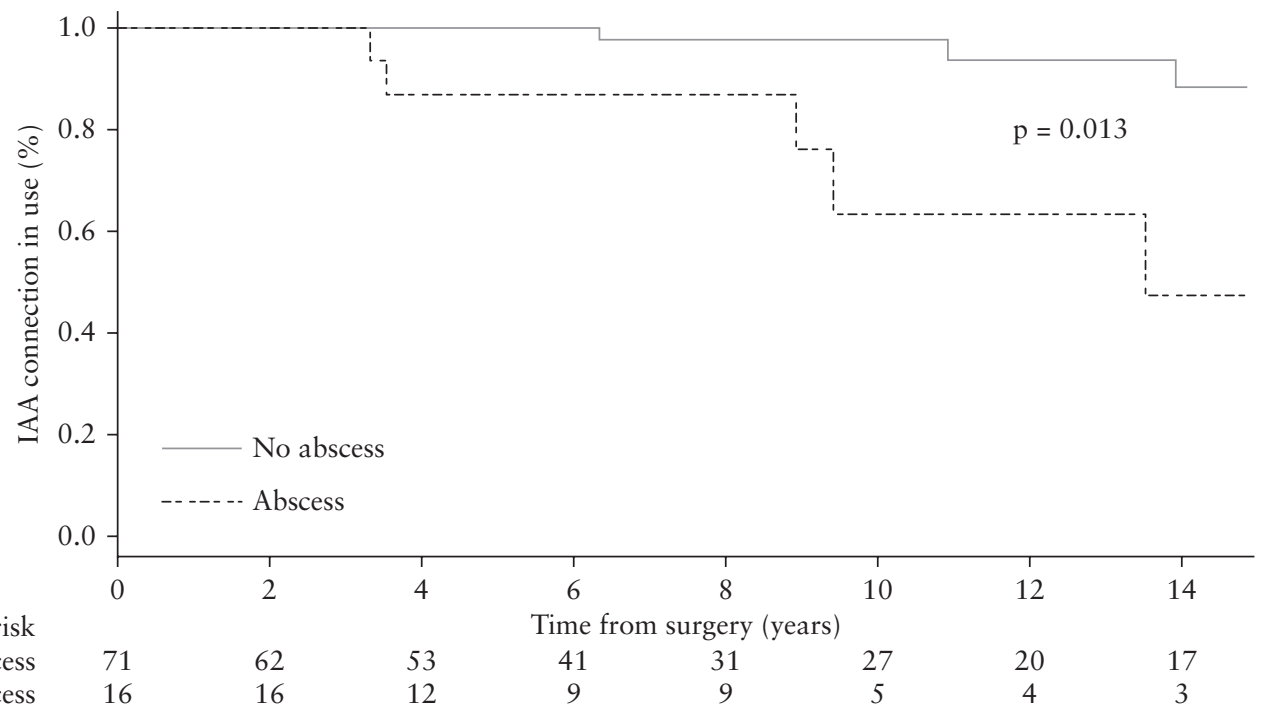

Figure 1. Kaplan-Meier survival curves of ileoanal anastomosis connection in use in paediatric patients with ulcerative colitis. A. Crohn's disease [CD] diagnosis $[p=0.005]$. B. Postoperative fistulas $[p=0.007]$. C. Postoperative abscesses [ $p=0.013]$; ${ }^{*} p$-values are calculated with a maximum follow-up of 10 years. 
Table 4. Bowel function at latest follow-up or before ileostomy

\begin{tabular}{|c|c|c|c|c|}
\hline & All patients & IAA still in use & IAA abandoned & $p$-Value \\
\hline Patients, $n[\%]$ & $87[100 \%]$ & $79[91 \%]$ & $8[9 \%]$ & \\
\hline Follow-up time [years] & $6.4[2.7-7.9]$ & $6.4[2.5-11.7]$ & $7.2[3.5-9.6]$ & 0.041 \\
\hline Daytime defaecation frequency & $5[4.5-7.5]$ & $5[4-7]$ & $9.3[7.5-10]$ & 0.004 \\
\hline Defaecation frequency at night & $1[0-1.5]$ & $0.5[0-1]$ & $2[1-2.6]$ & 0.007 \\
\hline Incontinence, $n[\%]^{\mathrm{a}}$ & & & & 0.042 \\
\hline No & $37[59 \%]$ & $37[62 \%]$ & 0 & \\
\hline Mild & $25[40 \%]$ & $22[37 \%]$ & $3[100 \%]$ & \\
\hline Severe & $1[2 \%]$ & $1[2 \%]$ & 0 & \\
\hline Pouchitis, $n[\%]^{\mathrm{b}}$ & & & & 0.454 \\
\hline No pouchitis & $27[43 \%]$ & $24[42 \%]$ & $3[50 \%]$ & \\
\hline Single or few episodes & $24[38 \%]$ & $23[40 \%]$ & $1[17 \%]$ & \\
\hline Chronic & $12[19 \%]$ & $10[18 \%]$ & $2[33 \%]$ & \\
\hline
\end{tabular}

Continuous data are given as median with interquartile range and categorical data as frequencies. Defaecation frequencies are patient-reported. Pouchitis was registered in symptomatic patients who were prescribed antibiotics during the year preceding the follow-up. Severe incontinence indicates uncontrolled defaecation, and mild incontinence is considered to be scarce or rare staining.

IAA, ileoanal anastomosis.

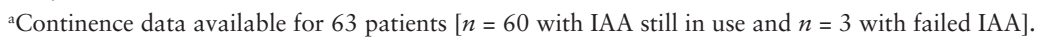

bouchitis data available for 63 patients [ $n=57$ with IAA still in use and $n=6$ with failed IAA].

colonic disease distribution, backwash ileitis, superficial fissures, and even transmural lymphoid aggregates. ${ }^{24,26,27}$ Importantly, the typical histopathological findings of CD may be missed if the diagnosis is solely based on mucosal biopsies, underlining the importance of appropriate differential diagnostics. ${ }^{24}$ In our sample, patients with later CD diagnosis were more likely to be operated in three stages, reflecting uncertainty of the diagnosis before surgery despite extended diagnostic work-up or a severe phase of the disease. This suggests that even full histopathological assessment of colonic specimens has limited additional value for identification of CD. A threestage procedure with separate colectomy is indicated when the patient presents acutely with severe symptoms such as toxic megacolon or uncertain diagnosis of UC, or when problems such as growth delay, steroid dependence, or malnutrition favour weight gain or tapering of corticosteroids before the IAA procedure. ${ }^{28}$ Some centres delay creation of the pouch after proctocolectomy until adolescence. ${ }^{21,29,30}$ However, the results obtained in our study among patients with retained ileoanal continuity support operative management during childhood unless the possibility of CD is of a special concern.

After proctocolectomy with IAA, as many as $45-75 \%$ of paediatric patients have at least one postoperative complication, ${ }^{5,8,17}$ which associates with worse long-term outcomes and impaired quality of life. ${ }^{5,7,8,23}$ Overall, our postoperative complication rate was similar compared with earlier studies. Fistulas and abscesses have been reported to occur in $10-30 \%$ and $12-35 \%$ of children after IAA, respectively, and their occurrence relates closely with postoperative CD diagnosis..$^{5-8,10,16,17}$ In our sample, the frequency of postoperative strictures and leakages was similar or lower than previously reported..$^{5-8,16,18}$ Interestingly, strictures and leakages were equally common among patients with UC or CD, and they did not influence the prognosis of the anastomosis, suggesting that such complications themselves do not worsen the outcome when managed appropriately. Similar to previous findings, ${ }^{5,17}$ reoperations particularly due to bowel obstructions were very common, occurring in almost half of our patients. However, the need for relaparotomy itself or the occurrence of postoperative bowel obstruction or haemorrhage did not influence the prognosis of the pouch. IBD patients appear highly susceptible to complications regardless of the type of surgical approach, since laparoscopic proctocolectomy has not been shown to decrease the risk for reoperations. ${ }^{31,32}$

After proctocolectomy with IAA during childhood, patients present with an average daily defaecation frequency of 4-7 and 1-2 during the night, ${ }^{5,6,16-18,20}$ as reported also among our patients. Both bowel movements and incontinence rates tend to increase in the long run, ${ }^{9}$ but functional outcomes among children still appear excellent after 15 years $^{9,17}$ [no longer follow-ups yet exist]. Severe incontinence is rare ${ }^{5,16,17}$ but relates to an increased pouch failure rate; also in our patients, those with later conversion to ileostomy presented with higher defaecation frequencies. Pouchitis episodes relate to worse functional outcomes and occur in $23-48 \%$ of patients after surgery. ${ }^{6,8,18}$ Over half of our patients had experienced clinical symptoms of pouchitis, but data were missing in a subset of children. We speculate that since functional outcomes are less likely registered among asymptomatic patients, the missing data may negatively distort our results. Chronic pouchitis has been previously found to increase the risk for pouch failure ${ }^{11,13}$ but was rare in our sample, and showed no associations with functional outcomes or pouch failure.

The primary limitation of our study is the retrospective data collection resulting in incomplete capture of pouchitis and continence data. On the other hand, the long and complete follow-up for the primary outcome measure pouch failure, inclusion of only children and not young adults, and monitoring the patients in the same institution where IAA surgery was carried out, are among the strengths of the present study, enabling a reliable analysis of the long-term outcomes.

In conclusion, postoperative $\mathrm{CD}$ is the most significant risk factor pouch failure after IAA. Need for three-stage surgery, and postoperative fistulas and abscesses associated with $\mathrm{CD}$, increased the risk for IAA failure. Differentiating UC from CD remains challenging, and despite extensive diagnostic work-up, in approximately one out of 10 patients the preoperative diagnosis will later be revised to CD. Among UC patients, postoperative complications occur at an acceptable frequency and they do not impair the prognosis of the pouch.

\section{Funding}

This work was supported by a research grant from the Sigrid Jusélius Foundation and the Pediatric Research Foundation. 


\section{Conflict of Interest}

There are no conflicts of interest to declare.

\section{Author Contributions}

IN, MH, K-LK, and MPP have participated in the study design. AK, IN, LM-S, K-LK, MPP, and RR have participated in data acquisition. IN, MH, and MPP are responsible for analysis of the data. All authors have revised the manuscript critically and have seen and agreed the final version of article to be submitted.

\section{References}

1. Virta LJ, Saarinen MM, Kolho KL. Inflammatory bowel disease incidence is on the continuous rise among all paediatric patients except for the very young: a nationwide registry-based study on 28-year follow-up. J Crohns Colitis 2017;11:150-6.

2. Samson C M, Escher J C. Corticosteroids. In: Baldassano RN, Grossman $\mathrm{AB}$, Kelsen JR et al., editors. Pediatric Inflammatory Bowel Disease. 3rd edn. New York, NY: Springer; 2017: 369-73.

3. Turunen P, Ashorn M, Auvinen A, Iltanen S, Huhtala H, Kolho KL. Longterm health outcomes in pediatric inflammatory bowel disease: a population-based study. Inflamm Bowel Dis 2009;15:56-62.

4. Mattei P. Surgical treatment of ulcerative colitis. In: Baldassano RN, Grossman AB, Kelsen JR et al., editors. Pediatric Inflammatory Bowel Disease. 3rd edn. New York, NY: Springer; 2017: 519-31.

5. Pakarinen MP, Natunen J, Ashorn M, et al. Long-term outcomes of restorative proctocolectomy in children with ulcerative colitis. Pediatrics 2009;123:1377-82.

6. Ozdemir Y, Kiran RP, Erem HH, et al. Functional outcomes and complications after restorative proctocolectomy and ileal pouch anal anastomosis in the pediatric population. J Am Coll Surg 2014;218:328-35.

7. Hirata A, Uchino M, Bando T, et al. Long-term outcomes and sex differences after restorative proctocolectomy in pediatric patients with ulcerative colitis. J Pediatr Surg 2016;51:454-60.

8. Alexander F, Sarigol S, DiFiore J, et al. Fate of the pouch in 151 pediatric patients after ileal pouch anal anastomosis. J Pediatr Surg 2003;38:78-82.

9. Polites SF, Potter DD, Moir CR, et al. Long-term outcomes of ileal pouchanal anastomosis for pediatric chronic ulcerative colitis. J Pediatr Surg 2015;50:1625-9

10. Shannon A, Eng K, Kay M, et al. Long-term follow up of ileal pouch anal anastomosis in a large cohort of pediatric and young adult patients with ulcerative colitis. J Pediatr Surg 2016;51:1181-6.

11. Fazio VW, Kiran RP, Remzi FH, et al. Ileal pouch anal anastomosis: analysis of outcome and quality of life in 3707 patients. Ann Surg 2013;257:679-85.

12. Michelassi F, Lee J, Rubin M, et al. Long-term functional results after ileal pouch anal restorative proctocolectomy for ulcerative colitis: a prospective observational study. Ann Surg 2003;238:433-41; discussion 442-5.

13. Yu CS, Pemberton JH, Larson D. Ileal pouch-anal anastomosis in patients with indeterminate colitis: long-term results. Dis Colon Rectum 2000;43:1487-96.
14. Belliveau P, Trudel J, Vasilevsky CA, Stein B, Gordon PH. Ileoanal anastomosis with reservoirs: complications and long-term results. Can J Surg 1999;42:345-52.

15. Braveman JM, Schoetz DJ Jr, Marcello PW, et al. The fate of the ileal pouch in patients developing Crohn's disease. Dis Colon Rectum 2004;47:1613-9.

16. Lillehei CW, Leichtner A, Bousvaros A, Shamberger RC. Restorative proctocolectomy and ileal pouch-anal anastomosis in children. Dis Colon Rectum 2009;52:1645-9.

17. van Balkom KA, Beld MP, Visschers RG, van Gemert WG, Breukink SO. Long-term results after restorative proctocolectomy with ileal pouch-anal anastomosis at a young age. Dis Colon Rectum 2012;55:939-47.

18. Knod JL, Holder M, Cortez AR, et al. Surgical outcomes, bowel habits and quality of life in young patients after ileoanal anastomosis for ulcerative colitis. J Pediatr Surg 2016;51:1246-50.

19. Mortellaro VE, Green J, Islam S, Bass JA, Fike FB, St Peter SD. Occurrence of Crohn's disease in children after total colectomy for ulcerative colitis. $J$ Surg Res 2011;170:38-40.

20. Wewer V, Hesselfeldt P, Qvist N, Husby S, Paerregaard A. J-pouch ileoanal anastomosis in children and adolescents with ulcerative colitis: functional outcome, satisfaction and impact on social life. J Pediatr Gastroenterol Nutr 2005;40:189-93.

21. Jones I, Ramani P, Spray C, Cusick E. How secure is the diagnosis of ulcerative colitis in children, even after colectomy? J Pediatr Gastroenterol Nutr 2018;66:69-72.

22. Reese GE, Lovegrove RE, Tilney HS, et al. The effect of Crohn's disease on outcomes after restorative proctocolectomy. Dis Colon Rectum 2007;50:239-50.

23. Abdelaal K, Jaffray B. Variables associated with loss of ileoanal pouches constructed in childhood. J Pediatr Surg 2017;52:281-5.

24. Yantiss RK, Odze RD. Diagnostic difficulties in inflammatory bowel disease pathology. Histopathology 2006;48:116-32.

25. Rosen MJ, Dhawan A, Saeed SA. Inflammatory bowel disease in children and adolescents. JAMA Pediatr 2015;169:1053-60.

26. Robert ME, Tang L, Hao LM, Reyes-Mugica M. Patterns of inflammation in mucosal biopsies of ulcerative colitis: perceived differences in pediatric populations are limited to children younger than 10 years. Am J Surg Pathol 2004;28:183-9.

27. Uchida K, Araki T, Hashimoto K, et al. Segmental distribution in refractory ulcerative colitis: a histological evaluation in pediatric and adult patients who underwent proctocolectomy. Inflamm Bowel Dis 2014;20:1227-35.

28. Bikhchandani J, Polites SF, Wagie AE, Habermann EB, Cima RR. National trends of 3- versus 2-stage restorative proctocolectomy for chronic ulcerative colitis. Dis Colon Rectum 2015;58:199-204.

29. Rialon KL, Crowley E, Seemann NM, Fahy AS, Muise A, Langer JC. Longterm outcomes for children with very early-onset colitis: implications for surgical management. J Pediatr Surg 2018;53:964-7.

30. Baillie CT, Smith JA. Surgical strategies in paediatric inflammatory bowel disease. World J Gastroenterol 2015;21:6101-16.

31. Fraser JD, Garey CL, Laituri CA, Sharp RJ, Ostlie DJ, St Peter SD. Outcomes of laparoscopic and open total colectomy in the pediatric population. J Laparoendosc Adv Surg Tech A 2010;20:659-60.

32. Diamond IR, Gerstle JT, Kim PC, Langer JC. Outcomes after laparoscopic surgery in children with inflammatory bowel disease. Surg Endosc 2010;24:2796-802. 